\title{
MAGNETIC FIELD DEPENDENCE OF SPIN-LATTICE RELAXATION ENHANCEMENT USING PIPERIDINYL NITROXYL SPIN-LABELS
}

\author{
J. D. Lovin, ${ }^{*}$ G. E. Wesbey, $\dagger$ B. L. Engelstad, $\dagger$ G. Sosnovsky, \\ M. Moseley,§ D. L. TuCK, †§ AND R. C. Brasch $\dagger$ \\ *Department of Radiology, University of Michigan, Ann Arbor, MI 48109, †Contrast Medium Laboratory, \\ Department of Radiology, School of Medicine, University of California, San Francisco, CA 94143, $¥$ The \\ Department of Chemistry, University of Wisconsin, Milwaukee, WI 53201, §The Department of Pharmaceutical \\ Chemistry, School of Pharmacy, University of California, San Francisco, CA 94143
}

\begin{abstract}
We examined the magnetic resonance properties of 12 paramagnetic piperidinyl nitroxyls in water and plasma solutions. Paramagnetic contributions to proton relaxation times were measured using 10.7 and $100 \mathrm{MHz}$ spectrometers. Proton relaxation enhancement from nitroxyls increased with ascending molecular weight, in plasma solutions versus equimolar aqueous solutions, and with measurements at $10.7 \mathrm{MHz}$ compared to $100 \mathrm{MHz}$. Relaxation rates were observed to approximately double at $10.7 \mathrm{MHz}$ compared to $100 \mathrm{MHz}$ and from water to plasma solutions. The data indicate that proton spin-lattice relaxation enhancement is magnetic field-dependent, and increases using nitroxyls of large molecular weight and with chemical substitutents that increase the microviscosity of solvent water molecules. The development of nitroxyls for diagnostic MRI will be aided by understanding these in vitro physical characteristics and trends.
\end{abstract}

Keywords: Nitroxyl spin labels, Magnetic resonance contrast media, Field-dependent relaxation

\section{INTRODUCTION}

Magnetic resonance imaging (MRI) has exciting potential as a diagnostic tool useful in clinical imaging of the human body. ${ }^{7,13,19.51}$ Contrast agents may augment diagnostic information from MRI. Numerous contrast agents have been proposed including lasix, ${ }^{2}$ perfluorocarbon emulsions, ${ }^{15}$ dilute oral iron solutions for gastrointestinal contrast, ${ }^{46}$ and rare-earth complexes; ${ }^{14.45}$ the last two categories of compounds are termed paramagnetic because they possess an electron with an unpaired spin. ${ }^{25.34 .42}$

The strength and time decay of the MRI signal depends on a tissue's hydrogen density, intrinsic longitudinal relaxation time $\left(T_{1}\right)$, intrinsic transverse relaxation time $\left(T_{2}\right),{ }^{30}$ and the translational self-diffusion coefficient. ${ }^{48,49}$ These relaxation times are influenced by the microscopic magnetic environment surrounding each proton. Because the combination of these properties varies among tissues, excellent inherent contrast in MRI is obtainable among bodily organs.
MRI is exquisitely sensitive to the detection of disease, but may be rather poor in its ability to specifically diagnose an abnormality. ${ }^{34}$ Diagnostic specificity may be improved with tissue-specific paramagnetic pharmaceuticals. Nitroxyl spin labels (NSLs) have potential as paramagnetic agents for MRI because of a chemically stable unpaired electron. When NSL are placed in a magnetic field, the electron aligns with the field; as a much stronger magnetic dipole than unpaired protons, the unpaired electron alters the local magnetic field experienced by protons around it, reducing $T_{1}$ and $T_{2}$ relaxation times (increasing relaxation rates, $1 / T_{1}, 1 / T_{2}$ ). This property is termed paramagnetic proton relaxation enhancement.

NSL are synthetic organic free radicals and are, as a class, potentially useful paramagnetic contrast agents for MRI. Like other paramagnetic substances, NSLs increase $T_{1}$ and $T_{2}$ relaxation rates of hydrogen nuclei, thus altering signal intensity. We report data that compare efficiency of proton relaxation enhancement
RECEIVED 8/6/84; ACCEPTED 12/2/84.

Address correspondence and reprint requests to Robert $C$. Brasch, M.D., Contrast Medium Laboratory, Department of Radiology, University of California, San Francisco, CA 94143.
Acknowledgement-Supported in part by NIH Grant AM 31937 from the National Institutes of Arthritis. Diabetes and Kidney Diseases and by Schering AG, Berlin, Germany. 
using a series of piperidinyl NSLs differing in size and substituents.

The experimental design compares proton spinlattice relaxation in water and plasma at two widely different, but clinically relevant, magnetic field strengths: $0.25 \mathrm{~T}$ and $2.3 \mathrm{~T}$. These two field strengths approximate "low-field" and "high-field" human MRI systems currently in use. We report a range of from 1.5- to 3.5- fold decrease in NSL-induced spin lattice relaxation rate enhancement comparing lowfield to high-field results.

These in vitro data from multiple concentrations of each NSL provide a foundation for in vivo testing of the more successful relaxing agents of these NSL, and indicate a trend of magnetic-field dependence of the proton spin-lattice relaxation enhancement using NSLs.

\section{MATERIALS AND METHODS}

\section{Chemicals:}

Twelve piperidinyl NSL compounds were either purchased commercially (Eastman Kodak) or synthesized in our (G.S.) laboratory. Chemical structures and molecular weights (171 to 687) are shown in Fig 1. All compounds have in common a six-membered ring and methyl substituted alpha-carbons surrounding a nitroxyl group. Substitutions of the basic piperidinyl ring included alcohol, amide, aliphatic, ether and carboxylic acid groups. For each compound 10, 1, 0.1 and $0.01 \mathrm{mM}$ solutions were prepared in normal human plasma (PL) and in deionized distilled water (AQ). The nitroxyl group has been shown to be stable over a wide range of $\mathrm{pH}$ using electron spin resonance (ESR). ${ }^{9}$ For comparison purposes, plasma and aqueous solutions of gadolinium-DTPA (Schering AG) were prepared in identical concentrations. Samples of unadulterated deionized water and normal plasma served as reference standards to determine diamagnetic contributions to relaxation rates of solutions.

\section{NMR spectroscopy}

The proton spin-lattice relaxation times, $T_{1}$, were measured for all $10 \mathrm{mM}$ samples in both aqueous and plasma solutions using $100 \mathrm{MHz}$ (Varian XL 100) and 10.7 MHz (Praxis II) NMR spectrometers. The remaining concentrations $(1,0.1$, and $0.01 \mathrm{mmol})$ of aqueous solutions were analyzed for $T_{1}$ on the 10.7 $\mathrm{MHz}$ spectrometer. A partial saturation recovery technique was employed to measure $T_{1}$ on the $10.7 \mathrm{MHz}$ spectrometer. $T_{1}$ relaxation times on the $100 \mathrm{MHz}$ spectrometer were determined by the inversion recovery method. Both 10.7 and $100 \mathrm{MHz}$ measurements were performed at $23^{\circ} \mathrm{C} \pm 1.5^{\circ} \mathrm{C}$. The diamagnetic contributions to relaxation rate (as determined for water and plasma standards) were subtracted from observed rates of NSL solutions to obtain the paramagnetic (p) contributions to $T_{1}{ }^{29}$

\section{ESR spectroscopy}

Electron spin resonance (ESR) spectroscopy was performed on a Varian E-104A spectrometer operating at a $9.51 \mathrm{GHz}$ resonant frequency. Solutions were contained within a $0.04 \mathrm{ml}$ flat cell which was placed into the microwave cavity. The modulation amplitude of the magnetic field sweep was 20 gauss at $100 \mathrm{KHz}$, and a microwave power of $20 \mathrm{~mW}$ was used to record the first derivative of the absorption mode. The rotational correlation time $\left(\tau_{r}\right)$ of the NSL was estimated from the peak-to-peak linewidth in gauss of the lowfield line on the first derivative absorption spectrum, using a conventional formula. ${ }^{39}$

\section{Magnetic resonance imaging}

All solutions placed in test tubes were imaged on a $3.5 \mathrm{kG}$ spin-echo MRI system, operating at a hydrogen resonant frequency of $15 \mathrm{MHz} .^{18}$ The effective aperture for imaging was $6.5 \mathrm{~cm}$. The imaging mode chosen yielded pixel sizes of $0.5 \times 0.5 \mathrm{~mm}(\mathrm{XY})$ on a $128 \times$ 128 matrix, and five contiguous transverse sections, each $4.4 \mathrm{~mm}$ thick, were sampled. Four separate spin-echo intensity images were obtained for each section, with each image containing echo delay times (TE) of 28 or $56 \mathrm{msec}$. Pulse repetition times (TR) of 0.5 or $2.0 \mathrm{sec}$ were utilized. Spin-echo signal intensity values, in arbitrary units of voltage, were calculated for selected regions of interest.

\section{Correlation times}

An important variable influencing the degree of proton relaxation enhancement is the dipolar electronnuclear correlation time, which describes the interaction time modulating translational diffusion of the NSL and water proton $\left(\tau_{d}\right)$, or the lifetime of a labile electron-nuclear complex $\left(\tau_{c}\right)$. The dipolar translational paramagnetic correlation time, $\tau_{d}$, was estimated using the original theory developed by Hubbard. ${ }^{27}$ (See Appendix). $\tau_{d}$, the diffusion constant of solvent water protons $(D)$, and distance of closest approach of protons to the paramagnetic center $(d)$ all influence the contribution of "outer sphere" (longrange diffusion-mediated) relaxation $\left(R_{o}\right)$ to spinlattice relaxation. The paramagnetic correlation time, $\tau_{c}$, was estimated by comparison of proton $T_{1}$ relaxation times at $100 \mathrm{MHz}$ versus the $10.7 \mathrm{MHz}$ frequen$\mathrm{cy}^{29}$ using conventional Solomon-Bloembergen (S-B) theory, ${ }^{5,43}$ which assumes formation of a labile electron-nuclear complex via dipolar coupling (see Appendix). 


\section{NSL STRUCTURES}

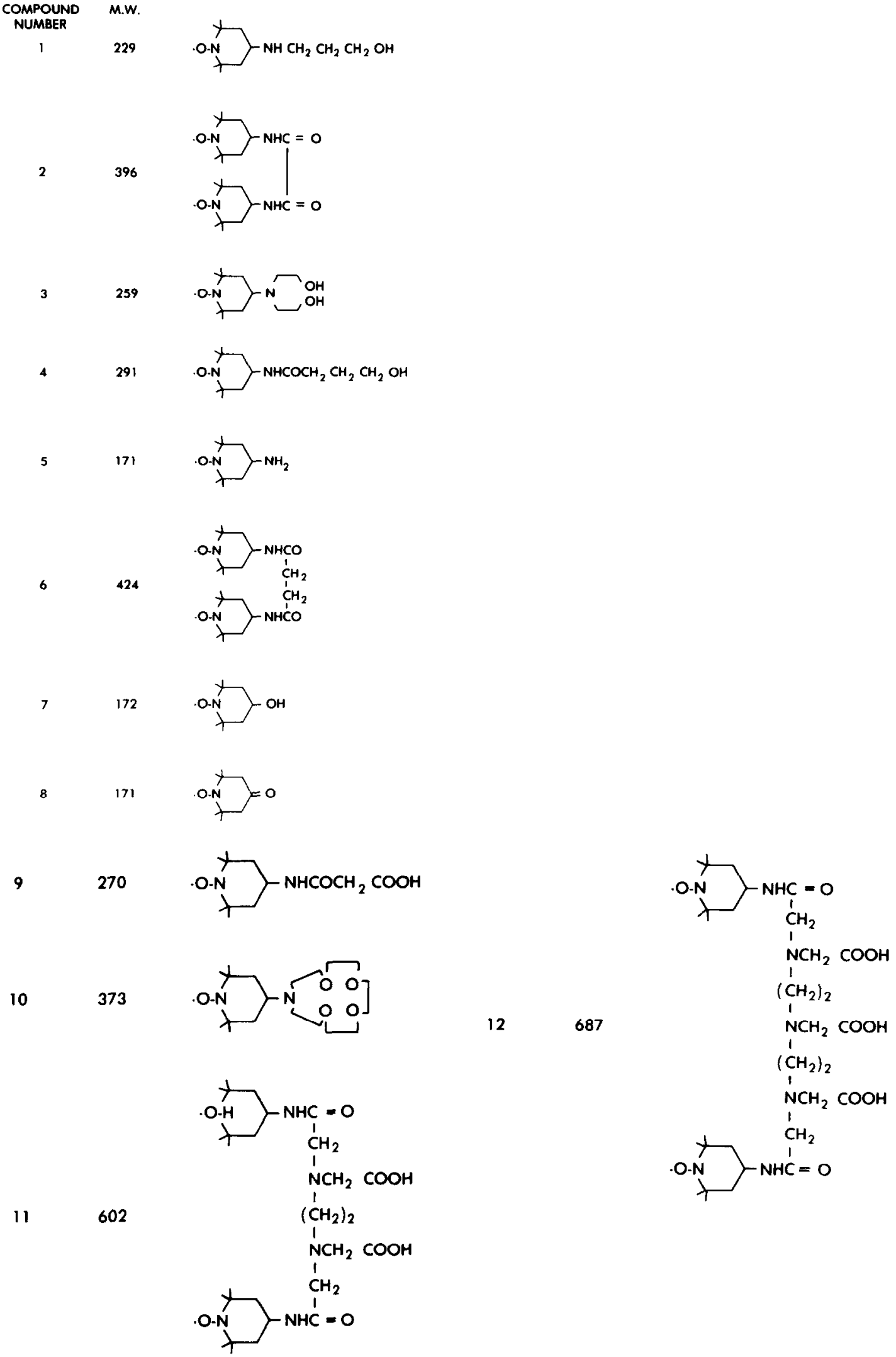

Fig. 1. Chemical structures of nitroxyl spin labels studied in this investigation. 
Table 1. Low field $1 / T_{1_{\mathrm{p}}}$ relaxation rates $\left(\mathrm{sec}^{-1}\right)$ for 10 $\mathrm{mM}$ solutions

\begin{tabular}{ccc}
\hline $\begin{array}{c}\text { Compound } \\
\text { Number }\end{array}$ & $\begin{array}{c}10.7 \mathrm{MHz} \\
1 / T_{\mathrm{I}_{\mathrm{p}}}(\mathrm{AQ})\end{array}$ & $\begin{array}{c}10.7 \mathrm{MHz} \\
1 / T_{\mathrm{l}_{\mathrm{p}}}(\mathrm{PL})\end{array}$ \\
\hline 1 & 4.550 & 5.155 \\
2 & 4.686 & 8.264 \\
3 & 3.959 & 4.255 \\
4 & 4.948 & 7.937 \\
5 & 5.467 & 6.25 \\
6 & 3.163 & 8.130 \\
7 & 4.873 & 5.465 \\
8 & 2.516 & 4.049 \\
9 & 5.297 & 2.513 \\
10 & 3.805 & 4.854 \\
11 & 7.092 & 15.15 \\
12 & 7.40 & 9.804 \\
$13^{*}$ & 75.19 & 69.93 \\
\hline
\end{tabular}

*Gd-DTPA

\section{RESULTS}

\section{Stability of NSL solutions}

The NSL compounds were stable in aqueous and plasma solutions as measured by reproducible $T_{1}$ relaxation time measurements (less than $10 \%$ variation) over a period of three weeks.

\section{Paramagnetic contribution to $T_{1}$ relaxation}

Paramagnetic contributions to proton relaxation rates $\left(1 / T_{1_{p}}\right)$ were tabulated (Tables 1 and 2 ) to disclose trends among the various NSL's tested. Increasing proton relaxation rates were obvious with increasing concentration of paramagnetic NSL. Overall, $1 / T_{l_{\mathrm{o}}}$ increased to approximately $5 \mathrm{sec}^{-1}$ for 10 mmol concentrations of paramagnetic nitroxyls in aqueous solution. For the $1 \mathrm{mmol}$ aqueous solutions, $1 / T_{\mathrm{t}_{\mathrm{p}}}$ increased to approximately $0.5 \mathrm{sec}^{-1}$. The $1 / T_{1}$ of the deionized diamagnetic water reference standard was $0.4 \mathrm{sec}^{-1}$. No measurable increase in relaxation rates was seen with lower (.1 and $.01 \mathrm{mmol}$ ) concentrations of nitroxyls.

$1 / T_{1}$ relaxation rate enhancement was noticeably more effective at $10.7 \mathrm{MHz}$ than at $100 \mathrm{MHz}$ for both plasma and aqueous solutions, and more effective in plasma compared to aqueous solutions for equimolar concentrations of NSL (see $T_{1_{p}}(100 \mathrm{MHz}) / T_{1_{p}}(10.7$ $\mathrm{MHz}$ ) in Table 2). In water, a mean 1.70 fold (SD + 0.17 ) increase in $1 / T_{\mathrm{p}_{\mathrm{p}}}$ relaxation rates was seen comparing low-field to high-field; in plasma, the mean increase was even higher $(1.96, \pm \mathrm{SD} 0.14)$. The mean increases in $1 / T_{1_{0}}$ relaxation rate enhancement in plasma versus equimolar aqueous solutions of NSL was $1.42( \pm$ S.D. 0.53).

Relaxivity improved with ascending molecular weight. For example, NSL 1 (molecular weight 229) had $1 / T_{1_{p}}$ relaxation rates much lower than NSL II (molecular weight 602) or NSL 12 (molecular weight 687) for both aqueous and plasma $10 \mathrm{mM}$ solutions. The $1 / T_{1_{\mathrm{p}}}$ aqueous relaxation rate for NSL 1 was 4.55 $\mathrm{sec}^{-1}$ while NSL 11 was $7.09 \mathrm{sec}^{-1}$ and NSL 12 was $7.40 \mathrm{sec}^{-1}$.

Perhaps a more important variable than molecular weight is the number of unpaired electron spins per molecule. Di-nitroxyls as opposed to mono-nitroxyls exhibited improved relaxivity, probably because the former have double the number of unpaired electrons capable of providing more proton relaxation enhancement. For example, NSL's 2, 11 , and 12 with $1 / T_{1}$. relaxation rates of $4.69 \mathrm{sec}^{-1}, 7.09 \mathrm{sec}^{-1}$, and 7.40 $\mathrm{sec}^{-1}$ were more efficient than chemically similar mono-nitroxyls such as NSL $1\left(1 / T_{\mathrm{I}_{\mathrm{p}}}=4.55 \mathrm{sec}^{-1}\right)$.

\section{Paramagnetic correlation times: Plasma versus aqueous solutions}

To understand how the various NSLs might function in vivo as proton relaxation enhancers, all the NSLs in $10 \mathrm{mM}$ concentrations were tested in plasma. In particular, it was helpful to determine if there were any differences in the estimated paramagnetic correlation time $\left(\tau_{d}\right.$ or $\left.\tau_{c}\right)$ of the NSLs between plasma and aqueous solutions. The $\mathrm{pH}$ on all plasma samples tested was between 7 and 7.5.

Using the translational diffusion model of Hubbard, ${ }^{27}$ (see Appendix) we estimate a range of aqueous $\tau_{d}$ for the NSLs of $10^{-10.5}$ to $10^{-11} \mathrm{sec}$ (see Table 2), in

Table 2. High Field $1 / T_{1_{p}}$ relaxation rates $\left(\sec ^{-1}\right)$ and correlation times $(\mathrm{sec})^{*}$

\begin{tabular}{ccccccc}
\hline Compound & $\begin{array}{c}100 \mathrm{MHz} \\
1 / T_{\mathrm{1}_{\mathrm{p}}}(\mathrm{AQ})\end{array}$ & $\begin{array}{c}100 \mathrm{mHz} \\
1 / T_{\mathrm{p}_{\mathrm{p}}}(\mathrm{PL})\end{array}$ & $\begin{array}{c}\text { Aqueous } \\
\frac{T_{\mathrm{l}_{\mathrm{p}}}(100 \mathrm{MHz})}{T_{\mathrm{p}_{\mathrm{p}}}(10.7 \mathrm{MHz})}\end{array}$ & $\begin{array}{c}\text { Estimated } \\
\text { Aqueous } \\
\tau_{d}(-\log , \mathrm{sec})\end{array}$ & $\begin{array}{c}\text { Plasma } \\
\frac{T_{\mathrm{p}_{\mathrm{p}}}(100 \mathrm{MHz})}{1 / T_{\mathrm{1}_{\mathrm{p}}}(10.7 \mathrm{MHZ})}\end{array}$ & $\begin{array}{c}\text { Estimated } \\
\text { Plasma } \\
\tau_{d}(-\log , \mathrm{sec})\end{array}$ \\
\hline 1 & 2.288 & 2.632 & 1.65 & 10.7 & 1.82 & 10.5 \\
2 & 2.826 & 2.336 & 1.66 & 10.7 & 3.54 & 10.0 \\
3 & 2.288 & 2.288 & 1.73 & 10.8 & 1.86 & 10.5 \\
$?$ & 2.112 & 2.865 & 1.50 & 10.5 & 2.84 & 10.2 \\
11 & 3.600 & 5.155 & 1.97 & 11.0 & 2.93 & 10.2 \\
\hline
\end{tabular}

*10 mM NSL concentration. 
general agreement with previous reports. ${ }^{21,23}$ These estimations of the diffusional correlational time were made by comparing $T_{1_{\mathrm{o}}}$ 's at $100 \mathrm{MHz}$ versus 10.7 $\mathrm{MHz}$, assuming that the electron-nuclear dipolar interaction modulating water proton relaxation is described by the relative translational diffusion of the water proton and the NSL electron.

Aqueous $\tau_{c}$ was estimated by the ratio of $T_{\mathrm{t}_{\mathrm{p}}}$ relaxation at $100 \mathrm{MHz}$ versus $10.7 \mathrm{MHz},{ }^{29}$ using the standard Solomon electron-nuclear dipolar equations, ${ }^{43}$ ignoring the scalar term of Bloembergen. ${ }^{s}$ For all aqueous solutions of nitroxyls, $\tau_{c}$ was estimated to be between $10^{-10.6}$ and $10^{-10.3}$ seconds. This estimate was based on the published value of aqueous $\tau_{r}$ (rotational correlation time) for NSL 5 (MW 171, $\log \tau_{r}=-10.4$ sec at $\left.20^{\circ} \mathrm{C}\right)^{39}$ and for NSL 8 (MW 171, $\log \tau_{r}-10.9$ sec at $\left.20^{\circ} \mathrm{C}\right)^{37}$, as well as our own ESR spectral calculations of $\tau_{r}$ on these NSL's. We assumed that $\tau_{r}$, not $\tau_{m}$, was the rate limiting correlation time for the Solomon-Bloembergen model of NSL-relaxation enhancement. These $\tau_{r}$ values led us to choose the longer of two possible $\tau_{\mathrm{c}}$ values at each value of the ratio of $T_{\mathrm{I}_{\mathrm{p}}}$ $(100 \mathrm{Mhz}) / T_{1_{\mathrm{o}}}(10.7 \mathrm{MHz})$ for the NSLs. Without these $\tau_{r}$ values, an unambiguous determination of aqueous $\tau_{c}$ would not be possible.

Applying the Hubbard diffusion-model to the plasma NSL solutions, we estimate $\tau_{d}$ to range from $10^{-10}$ to $10^{-10.5} \mathrm{sec}$. This is in reasonable agreement with our calculation of $\tau_{d}$ from the NSL-protein dispersion studies of Polnaszek and Bryant $\left(10^{-9.9} \mathrm{sec}\right),{ }^{41}$ which was performed over a wide range $(0.01 \mathrm{MHz}$ to $30 \mathrm{MHz}$ ) of numerous different proton resonant frequencies on a field-cycling spectrometer. Thus, we would conclude that the microviscosity of water protons in plasma diffusing near the nitroxyl paramagnetic center is higher (slower diffusion) than in pure water, a conclusion supported by pulsed-gradient spinecho NMR translational self-diffusion measurements of Cooper, et al. ${ }^{16}$ In that study, the diffusivity ratio of bulk water in human plasma versus pure water was found to be 0.86 . Polnaszek and Bryant ${ }^{41}$ found much slower water self-diffusion at the surface (defined as the first several monolayers of water) of their spinlabelled protein. Their diffusivity ratio of surface water on the spin-labelled protein relative to an aqueous spin label solution was 0.19 .

In plasma, at least three nitroxyls emerged as having much longer estimated $\tau_{c}$ 's, down to $10^{-8.5}$ to $10^{-8.6} \mathrm{sec}$ (NSL 2, 6, 11). These estimations of exchange correlation times were measured by comparing nitroxyl paramagnetic $T_{\mathrm{I}_{\mathrm{p}}}$ 's at $100 \mathrm{MIIz}$ versus $10.7 \mathrm{MHz}{ }^{29}$ The data on these three nitroxyls show that $T_{1}$ decreased to an extent that the paramagnetic relaxation rate enhancement ratio $(E)$ roughly doubled at $10.7 \mathrm{MHz}$
(Table 1), comparing the ratio of $10 \mathrm{mM} 1 / T_{1_{\mathrm{p}}}$ in plasma NSLs to $10 \mathrm{mmol} 1 / T_{1_{\mathrm{r}}}$ aqueous NSLs. We were only able to assign a single, unambiguous $\tau_{c}$ to a NSL in plasma if the ratio of $T_{l_{p}}(100 \mathrm{MHz}) / T_{1_{p}}(10.7$ $\mathrm{MHz}$ ) exceeded a minimum value of 2.6. This value (2.6) was obtained from the peak $T_{I_{\mathrm{p}}}$ ratio calculated from the Solomon equation for a range of $\tau_{c}$ 's $\left(10^{-12}\right.$ to $10^{-10} \mathrm{sec}$ ). For the nine NSLs where high field and low field $10 \mathrm{mM}$ plasma $T_{1_{\mathrm{p}}}$ 's were measured, the mean ratio was $1.96, \mathrm{SD} \pm 0.14$, range 1.82 to 2.18 . For $T_{1_{\mathrm{r}}}$ $(100 \mathrm{MHz}) / T_{\mathrm{I}_{\mathrm{p}}}(10.7 \mathrm{MHz})$ ratios of less than 2.6, three different $T_{c}$ values are possible.

Electron spin resonance spectroscopy of these three NSL's $(2,6,11)$, plus two other NSL's $(4,5)$ in water and plasma failed to demonstrate any lengthening of $\tau$, from water to plasma. ESR spectra are very sensitive indicators of the rotational molecular motion of the NSL. ${ }^{4}$ The absence of any lengthening of $\tau_{r}$ from water to plasma for all NSL studied by ESR is a strong argument against the use of the Solomon-Bloembergen exchange model for NSL-induced water proton relaxation enhancement, especially in plasma. Since $\tau_{r}$ (determined by FSR) for the NSI in plasma was the same as in water $\left(\tau_{r}=10^{-10.4}\right.$ to $\left.10^{-10.9} \mathrm{sec}\right), \tau_{c}$ in plasma can be no longer than $10^{-10.4} \mathrm{sec}$. Thus, a model which calculates $\tau_{c}$ for three NSL at $10^{-8.5}$ to $10^{-8.6} \mathrm{sec}$ must be considered inadequate. Our data thus supports the consensus in the literature ${ }^{20,22,24,27,28,35.40,41}$ that NSLinduced solvent proton relaxation is predominately modulated by the relative translational self-diffusion of water protons near the NSL's unpaired electron.

\section{MRI signal intensity}

Signal intensity data observed for the NSL solutions showed that maximal relaxation enhancement did not correspond with maximal MRI signal intensity (Table 3). Because spin-echo MRI depends on both $T_{1}$ and $T_{2}$ relaxation times, $T_{1}$ shortening generally acts to increase image intensity: however, $T_{2}$ shortening also observed with paramagnetics usually acts to decrease signal intensity. ${ }^{46}$ The observed MRI signal intensity is highly dependent on the TR and TE values chosen. A paramagnetic that preferentially shortens $T_{1}$ over $T_{2}$ will have the highest signal intensity, but could conceivably be considered a relatively inefficient proton relaxation enhancer. In particular, at pulse repetition times of $500 \mathrm{~ms}$, NSL 11 was found to have the highest MRI signal intensity. In fact, several nitroxyls had greater signal intensity at $10 \mathrm{mM}$ concentrations than gadolinium-DTPA - another MRI contrast agent. ${ }^{45,47}$ This occurred because gadolinium-DTPA's relaxation enhancement dominates at this concentration, producing decreased spin-echo signal intensity. 
Table 3. MRI signal intensities

\begin{tabular}{|c|c|c|c|c|}
\hline \multirow{2}{*}{$\begin{array}{l}\text { Com- } \\
\text { pound }\end{array}$} & \multicolumn{2}{|c|}{$\begin{array}{c}\text { MRI Intensity } \\
(\mathrm{TR}=500 \mathrm{msec}, \mathrm{TE}=28 \\
\text { msec })\end{array}$} & \multicolumn{2}{|c|}{$\begin{array}{l}\text { Rank order of } \\
\text { intensities }\end{array}$} \\
\hline & $10 \mathrm{mM}$ Conc. & $1 \mathrm{mM}$ Conc. & \multicolumn{2}{|c|}{$(\mathrm{TR}=500 \mathrm{msec})$} \\
\hline 1 & 11056 & 5908 & $10 \mathrm{mM}$ & $1 \mathrm{mM}$ \\
\hline 2 & 5902 & 3816 & 11 & 6 \\
\hline 3 & 4796 & 4423 & 10 & 9 \\
\hline 4 & 5642 & 3518 & 12 & 5 \\
\hline 5 & 14378 & 6177 & 9 & 1 \\
\hline 6 & 11988 & 7183 & 5 & 12 \\
\hline 7 & 11128 & 5216 & 6 & 10 \\
\hline 8 & 11722 & 5210 & 8 & 11 \\
\hline 9 & 14561 & 6335 & 7 & 7 \\
\hline 10 & 19928 & 5852 & 1 & 8 \\
\hline 11 & 19998 & 5544 & 2 & 3 \\
\hline 12 & 19690 & 5893 & 4 & 2 \\
\hline & & & 3 & 4 \\
\hline
\end{tabular}

${ }^{a}$ in arbitrary units of mean pixel voltage

\section{DISCUSSION}

The $1 / T_{\mathrm{I}_{\mathrm{p}}}$ relaxation rate enhancement observed from NSLs was noticeably more effective at $10.7 \mathrm{MHz}$ than $100 \mathrm{MHz}$, and in plasma compared to water. Our data does not permit a complete description of the magnetic field dependence for NSL-induced solvent $1 / T_{1_{p}}$ relaxation rate enhancement. Precise characterization of solvent water outer sphere diffusion contributions to $T_{\mathrm{I}_{\mathrm{p}}}$ would be best accomplished using fieldcycling spectrometer measurements of $T_{1_{\mathrm{p}}}$ relaxivity at multiple magnetic field strengths ("dispersion" studies) and temperatures, with subsequent quantitative analysis in light of various theories of outer sphere relaxation. ${ }^{24,27,28,40}$ The clinically relevant result reported herein is that NSL-induced $T_{1}$ water protonrelaxation rate enhancement is greater at lower MRI field strengths, with more dispersion between fields than reported for other potential MRI contrast agents including Mn-DTPA and gadolinium-DTPA. ${ }^{31}$ Our in vitro measurements of the $T_{1}$, relaxivity of aqueous Gd-DTPA at $10.7 \mathrm{MHz}$ and $100 \mathrm{MHz}$ showed nearidentical $T_{1_{p}}$ 's.

NSL's are not new to biophysical research and have been used for two decades as "spin-labels" for in vitro biological studies. ${ }^{44}$ Brasch and others have reported on a water soluble piperidinyl NSL derivative which is rapidly excreted in the urine after intravenous injection and provides physiological data on renal excretion by MRI urography. ${ }^{8-10}$ These investigators further point out that NSL's could serve to differentiate iso-intense abnormal tissues (e.g. tumor) from adjacent normal tissues. Paramagnetic contrast agents have potential to aid in assessments of capillary perfusion and organ function by paramagnetic labeling of cells and tissue-specific biomolecules. ${ }^{10,44}$ In addition,
NSLs can help to assess the integrity of the bloodbrain barrier. ${ }^{10}$ NSLs have also been used in the in vitro study of drug metabolism by covalent binding to drugs such as steroids. ${ }^{3}$

Toxicity tests of two prototype NSL contrast agents for possible mutagenesis and carcinogenesis have been performed. A modern and sensitive assay for chemical carcinogens is sister-chromatid exchange testing. ${ }^{50}$ Using this method, no evidence of mutagenesis for nitroxyls or their reduced metabolites was found.' Furthermore the $\mathrm{LD}_{50}$ in rats $(>15 \mathrm{mmol} / \mathrm{kg})$ has been demonstrated to be greater than 100 times the lowest effective dose for renal enhancement on spin echo images $(0.14 \mathrm{mmole} / \mathrm{kg})$.

The NSLs tested were not prone to spontaneous in vitro reduction. The measurements of proton relaxation times in water and plasma were reproducible over a period of three weeks on both the MR imager and the MRI spectrometer. The nitroxyl moiety itself is relatively stable. For example, it remains unchanged when heated up to $123^{\circ} \mathrm{C}$ and over a $\mathrm{pH}$ range of $1.7-10$. It can be stored at room temperature as a solid for 60 days or longer without loss of activity as documented by ESR spectroscopy. ${ }^{9}$ Yet, studies have shown that nitroxyls are quire susceptible to partial in vivo chemical reduction, perhaps from ascorbic acid or enzymes. ${ }^{17.26}$ Despite in vivo bioreduction, effective MRI contrast enhancement occurs. ${ }^{8-10}$

In plasma, NSL demonstrated definite lengthening of the dipolar diffusional correlation time $\left(\tau_{d}\right)$ in association with improved proton relaxation enhancement. This outer sphere diffusional paramagnetic correlation time, $\tau_{d}$, was lengthened from water to plasma in all the NSL's. As noted in the Appendix, the preponderance of previous work in the literature with NSL-solvent relaxation rate enhancement would favor an outer sphere solvent diffusion mechanism for the nitroxyl-water interaction. ${ }^{11,20-24,27,28,35,40,41}$

The importance of the NSL paramagnetic correlation, $\tau_{d}$, in effecting proton relaxation enhancement is not only suggested by our in vitro studies in plasma, but can also be inferred from previous in vivo MRI studies with NSL's. Brasch et al. ${ }^{10}$ found dramatic $T_{1}$ shortening induced by NSL 9 on a $15 \mathrm{MHz}$ MRI system in vivo for a canine cerebral abscess despite local ESR-determined NSL tissue concentrations of only $0.45 \mathrm{mM}$. In water, a $1 \mathrm{mM}$ concentration of NSL 9 shows very little $T_{1}$ shortening ( $T_{\mathrm{l}_{\mathrm{p}}}$ equals $1666 \mathrm{~ms}$, $T_{1}$ of water equals $2500 \mathrm{~ms}$ ). Thus, we may conjecture that in the tissue milieu in vivo, long paramagnetic correlation times, presumably resulting from NSLwater-macromolecular interactions, can enhance proton relaxation much greater than in a pure aqueous milieu. For instance, the rotational correlational time $\left(\tau_{r}\right)$ of a low molecular weight NSL bound to bovine 
brain hexokinase has been measured by ESR at a value of $4.2 \times 10^{-9}$ sec. $^{38}$ However, water translational diffusion is more important than the rotational tumbling rate of the NSL in determining NSL relaxivity. ${ }^{41}$ The microviscosity of water in biological tissues, as measured by the translational molecular self-diffusion coefficient, is at least $50 \%$ less than free water. ${ }^{16}$ Our studies would also lead to a prediction that in vivo fluid or tissue PRE on a $100 \mathrm{MHz}$ MRI system could be less dramatic than on a $10.7 \mathrm{MHz}$ system, for equivalent concentrations of NSL's, depending on the degree of diamagnetic $T_{1}$ lengthening in the tissue of interest at the higher field.

\section{CONCLUSION}

In summary, $T_{1}$ relaxation-rate enhancement of solvent water protons by nitroxyl spin labels is dependent on the static magnetic field strength. NSLs that have large molecular weights and interact with slowerdiffusing water molecules near plasma macromolecules cause greater proton relaxation rates than equimolar concentrations of small molecular weight aqueous NSL's. This is due to slowing of the correlation time, from slower solvent water translational self-diffusion. In addition, dinitroxyls without ESR evidence of strong electron spin-spin interaction had better relaxation enhancement. Unlike certain other paramagnetic metal or rare earth complexes, nitroxyl spin-labels can have variously substituted configurations to influence their biodistribution and thereby enhance their clinical utility.

\section{APPENDIX}

In a solution of NSL in water or in plasma, the nuclear spin of solvent water protons will be relaxed by one of two possible interactions with the unpaired electron on the NSL. The first interaction is formation of a labile molecular complex between the nuclear spin and electron spin via electron-nuclear dipolar coupling (Term A below) or scalar (contact interactions (Term $B$ below). The effectiveness of paramagnetic relaxation enhancement will then depend on many variables including the magnetic moment, electron-nuclear distance, field strength, and paramagnetic correlation time, $\tau_{c}$. Relaxation rates $\left(1 / T_{1_{M}}\right)$ of a proton in the vicinity of an unpaired electron are described by the Solomon-Bloembergen ${ }^{43,5}$ equation outlined below, which assume formation of a transient electronnuclear complex:

$$
\begin{aligned}
\frac{1}{T_{1 M}}= & \frac{2}{15} \frac{S(S+1) \gamma^{2} g^{2} \beta^{2}}{r^{6}}\left(\frac{3 \tau_{c}}{1+\omega_{1}^{2} \tau_{c}^{2}}+\frac{7 \tau_{c}}{1+\omega_{s}^{2} \tau_{c}^{2}}\right) \\
& +\frac{2}{3} \frac{S(S+1) A^{2}}{\hbar^{2}}\left(\frac{\tau_{e}}{1+\omega_{s}{ }^{2} \tau_{e}^{2}}\right)
\end{aligned}
$$

where $S$ is the electron spin quantum number; $g$ is the electronic $g$ factor; $\beta$ is the Bohr magneton; $\omega_{l}$ and $\omega_{\mathrm{S}}$ $\left(=657 \omega_{l}\right)$ are the Larmor angular precession frequencies for the nuclear spins and electron spins, respectively; $r$ is the ion-nucleus distance; $A$ is the hyperfine coupling constant; and $\tau_{\mathrm{c}}$ and $\tau_{\mathrm{e}}$ are the correlation times for the dipolar and scalar interactions, respectively, where $1 / \tau_{c}=1 / \tau_{r}+1 / T_{s}+1 / \tau_{m} . \tau_{r}$ is the rotational correlation time of the vector between the unpaired electron and the nuclear spin, ${ }^{36} T_{s}$ is the electron spin relaxation time, and $\tau_{m}$ is the lifetime of the nuclear spin in the sphere of influence of the paramagnetic center. Based on the relaxation data and conclusions reached by Endo et al., ${ }^{23}$ NSL-induced solvent proton relaxation is governed predominately by dipolar coupling (Term $A$ ), and scalar contributions (Term $B$ ) can be ignored.

If $\tau_{c}$ equals the reciprocal of the nuclear precessional frequency, $T_{1}$ relaxation rate enhancement is maximal (i.e. $1 / \tau_{c}$ increases with more optimal $\tau_{c}{ }_{c}$ 's). ${ }^{29}$ To increase $\tau_{c}$, one must know its limiting variable. Nitroxyls possess naturally long electron spin relaxation times (spin lattice relaxation time, $T_{1_{\mathrm{c}}}=10^{-5} \mathrm{sec}$; electron spin-spin relaxation time, $T_{2_{\mathrm{e}}}=10^{8} \mathrm{sec}$ ) which are at least 2 orders of magnitude longer than any other paramagnetic metal or lanthanide ion. ${ }^{21}$ The literature ${ }^{37,39}$ and our ESR measurements have calculated a $\tau_{r}$ of approximately $10^{-11}$ seconds for the low molecular weight NSLs currently undergoing evaluation as prototype MRI contrast agents. Thus, either $\tau$, or $\tau_{m}$ could be a limiting variable in determining $\tau_{c}$ for NSLs. Because $T_{s}$ is long at $10^{-8} \mathrm{sec}$, one could theoretically manipulate $\tau_{r}$ or $\tau_{m}$. To lengthen nitroxyls' $\tau_{r}$ beyond $10^{-11}$ seconds, and since $T_{s}$ can only get longer at higher magnetic fields, one must increase their molecular weight and/or bind NSLs to macromolecules. This will slow the rate at which nitroxyls tumble within solution. This may increase the proton relaxation rate, if $\tau_{m}$ is not the limiting variable (assume $\tau_{m}>10^{-11}$ seconds). Thus, proton relaxation enhancement could conceivably improve as $\tau_{c}$ approaches the reciprocal of the nuclear processional frequency, if one accepts the fundamental SolomonBloembergen assumption of formation of a labile electron-nuclear dipolar complex. Endo et al. ${ }^{23}$ point out that their investigation of NSL-solvent relaxationdispersion supported the application of SolomonBloembergen theory when proton-donor solvents were used. Certainly, water is a proton donor but they did not specifically study water as a solvent for NSL $T_{1}$ relaxation-enhancement. In theory, the ability to form such a labile electron-nuclear complex may be hampered by the common structural feature of NSLs, where four bulky methyl groups sterically hinder access of water to the unpaired electron on the nitroxyl 
moiety. More importantly, experimental data ${ }^{20,22,41}$ has indicated the inadequacy of the Solomon-Bloembergen model in describing NSL-induced solvent relaxation.

Thus, a second approach to understanding solvent proton-NSL electron interactions has evolved. These theories of Hubbard ${ }^{27}$ and Freed ${ }^{24,28}$ (based on earlier principles of outer sphere relaxation established by Bloembergen, Purcell, and Pound, ${ }^{6}$ and $\mathrm{Pfeiffer}^{40}$ ) assume that no chemical interaction between these two spins occurs. Rather, the electron-nculear dipolar interaction modulating water proton relaxation is described by the relative translational diffusion of the water proton and the NSL electron. The outer sphere relaxation process is characteristically seen with inaccessible "buried" paramagnetic centers, where a long range magnetic interaction between diffusing solvent water protons and the paramagnetic center induces fluctuations in the local magnetic field seen by the proton. ${ }^{32,33}$ One could regard the paramagnetic center of nitroxyls as being "buried", both by the stericallyhindering bulky methyl moieties on the organic ring, as well as by delocalization of the unpaired electron onto the nitrogen atom, making outer sphere effects important. Since the translational molecular self-diffusion coefficient of water is much greater than that of the NSL, the proton relaxation-enhancement induced by NSL is felt to be predominately dependent on water translational motion. ${ }^{11}$ In fact, Polnaszek and Bryant ${ }^{41}$ have measured the translational self-diffusion coefficient of water on the surface of a nitroxyl-protein, applying the force free diffusion model of Freed ${ }^{24,28}$ to $T_{1}$ measurements of NSL-protein complex in solution over a wide range of magnetic fields. Thus, for MRI, the local microviscosity of water in the various biological tissucs and fluids may well determine the magnetic field dependence of NSL-induced proton-relaxation enhancement. ${ }^{11}$ Now that we have developed a method for water self-diffusion measurements in MRI, ${ }^{48,49}$ this theory could eventually be tested in vivo.

Scalar coupling of free radical electrons with solvent nuclei is important with nuclei other than protons, such as ${ }^{13} \mathrm{C},{ }^{19} \mathrm{~F}$, and ${ }^{31} \mathrm{P} .{ }^{20}$ With these nuclei, both diffusion models and "sticking" models have been used to describe the scalar interaction.

\section{REFERENCES}

1. Avzal, V.; Brasch, R.C.; Nitecki, D.; Wolff, S. Nitroxyl spin label contrast enhancers for magnetic resonance imaging: Studies of acute toxicity and mutagenesis. Investigative Radiology 19:549-552, 1984.

2. Beall, P.T. Safe contrast agents for improved NMR contrast. Physiological Chemistry and Physics and Medical NMR 16:129-136, 1984.

3. Benson, W.R., Maienthal M. Synthesis of spin-labeled nitroxyl ester of steroids. Journal of Medicinal Chemistry 20:1308-1312;1977.

4. Blanchard, J.; Tozer, T.N.; Sorby, D.L.; et al. Investigation of drug-albumin interactions using spin-labeled bovine serum albumin. Molecular Pharmacology $11: 133-143 ; 1975$.

5. Bloembergen N. Proton relaxation times in paramagnetic solutions. J. Chem Phys 27:572-573; 1957.

6. Bloembergen, N.; Purcell, E.M.; Pound, R.V. Relaxation effects in nuclear magnetic resonance absorption. Phys. Rev. 70:460-474; 1948.

7. Brant-Zawadzki, M.; Davis, P.L.; Crooks, L.E.; et al. MRI in the visualization of cerebral abnormalities: Comparison with CT. AJR 4:117-124; 1983.

8. Brasch, R.C.; Ehman, D.L.; Wesbey, G.E.; et al. NMR tumor enhancement using a new paramagnetic pyrrolidine contrast agent. Radiology 149(P):99; 1983 (Abstract).

9. Brasch, R.C.; London, D.; Wesbey, G.E.; et al. Work in progress: Nuclear magnetic resonance study of a paramagnetic nitroxide contrast agent for enhancement of renal structures in experimental animals. Radiology 147:773-779; 1983.

10. Brasch, R.C.; Nitecki, D.E.; Brant-Zawadski, M.; Enzmann, D.R.; Wesbey, G.E. Brain NMR imaging enhanced by a paramagnetic contrast agent: Preliminary report. $A J R$ 141:1019-1023; 1983.

11. Bryant, R.G.; Polnaszek, C.F.; Kennedy, S.D.; et al. The magnetic field dependence of water proton $T_{1}$ in aqueous nitroxidc solutions: Implications for imaging contrast media (manuscript submitted).

12. Burkett, D.J.; Dwek, R.A.; Radda, G.K.; Richard, R.E.; Salmon, A.J. Probes for the conformational transitions of phosphorylase b. European Journal of Biochemistry 20:494-508; 1981 .

13. Bydder, G.M.; Steiner, R.E.; Young, I.R.; et al. Clinical NMR imaging of the brain: 140 cases. AJR 139:215$236 ; 1982$.

14. Caille, J.M.; et al. Gadolinium as a contrast agent in NMR. AJNR 5:1041-1042; 1983.

15. Clark, L.C.; et al. High contrast tissue and blood oxygen imaging based on fluorocarbon 19-F NMR relaxation times. In: Society of Magnetic Resonance in Medicine. 2nd Annual Meeting, San Francisco, 1983. (Abstract). Magnetic Resonance in Medicine 1:135-136; 1984.

16. Cooper, R.L.; Chang, D.B.; Young, A.C.; et al. Restricted diffusion in biophysical systems. Biophys. $J$. 14:161-177, 1974

17. Couet. W.R., Eriksson, U.G.; Tozer, T.N.; Tuck, L.D.; Wesbey, G.E.; Nitecki, D.; Brasch, R.C. Pharmacokinetics and metabolic fate of two nitroxides potentially useful as contrast agents for magnetic resonance imaging. Pharmaceutical Research (in press).

18. Crooks, L.E.; Hoenninger, J.; Arakawa, M., et al. Tomography of hydrogen with nuclear magnetic resonance. Radiology 136:701-706; 1980.

19. Doyle, R.H.; Pennock, J.M.; Banks, L.M.; et al. Nuclear magnetic resonance imaging of the liver. Initial experience. $A J R$ 138:193-200; 1982.

20. Dwek, R.A.; Hill, H.D.W.; Kenworthy, J.G.; Natusch, D.F.S.; Richards, R.E. Dynamic polarization of protons and fluorine nuclei in solutions of free radicals. Mol. Phys. 13:27, 1967. 
21. Dwek, R.A. Nuclear magnetic resonance (NMR) in biochemistry: applications to enzyme systems, chapters 9-12. New York: Oxford University Press, 1974.

22. Dwek, R.A.; Richards, R.E.; Taylor, D. Nuclear electron double resonance in liquids. In: Mooney, E.F.; ed. Ann. Rev. of NMR Spectroscopy 2:293-345, 1969.

23. Endo, K.; Morishima, I.; Yonezawa, T. Use of a stable free radical as a NMR spin probe for studying intermolecular interactions. XIV. A proton relaxation study of the hydrogen bond involving a stable-free radical. $J$. Chem. Phys. 67:4760-4767;1977.

24. Freed, J.H. Dynamic effects of pair correlation functions on spin relaxation by translational diffusion in liquids. II. Finite jumps and independent $T_{1}$ processes. $J$. Chem. Phys. 68:4034-4037; 1978.

25. Glasstone, S. Textbook of physical chemistry. 2nd ed. Toronto: D. Von Nostrand, 609-622; 1946.

26. Griffith, L.I.C.; Roben, G.M.; Rauckman, E.J.; Dreyer, B.P. Pharmacokinetics of nitroxide MRI contrast agents. In: Society of Magnetic Resonance in Medicine, 2nd Annual Meeting, San Francisco, CA, 1983, (Abstract). Magnetic Resonance in Medicine 1:159-160; 1984.

27. Hubbard, P.S. Theory of electron-nuclear Overhauser effects in liquids containing free radicals. Proc. Roy. Soc. (London) A291:537-555; 1966.

28. Hwang, L.P.; Freed, J.H. Dynamic effects of pair correlation functions on spin-relaxation by translational diffusion in liquids. J. Chem. Phys. 63:4017-4025; 1975.

29. James, T.L. Nuclear magnetic resonance in biochemistry: Principles and applications, New York: Academic Press, pp. 38-40, 46-48, 177-211; 1975.

30. Kaufman, L.; et al. Nuclear magnetic resonance imaging in medicine. New York: Igaku-Shoin, 1-240; 1981.

31. Koenig, S.H.; Baglin, C.; Brown, R.P.; Brewer, C.F. Magnetic field dependence of solvent proton relaxation induced by $\mathrm{Gd}+3$ and $\mathrm{Mn}+2$ complexes. Magnetic Resonance in Medicine 1(4):496-501, 1984.

32. Koenig, S.H.; Brown, R.D. Anomalous relaxation of water protons in solutions of copper containing proteins. Ann. NY Acad. Sci. 222:752-763, 1973.

33. Koenig, S.H.; Brown, R.D.; Lindstrom, T.R. Interactions of solvent with the heme region of methemoglobin and fluoro-methemoglobin. Biophys. J. 34:397-408, 1981.

34. Koutcher, J.A.; Burt, C.T.; Lauffer, R.B.; Brady, T.J. Contrast agents and spectroscopic probes in NMR. $J$. Nucl. Med. 25:506-513; 1984.

35. Kramer, K.D.; Muller-Warmuth, W.; Schindler, J. Molecular motion and relaxation in free-radical solutions of benzene, toluene, and some ethers as studied by dynamic nuclear polarization. $J$. Chem. Phys, 43:31-43, 1965.

36. Krugh, T.R. Spin-label induced nuclear magnetic resonance studies of enzymes. In Berliner, L.J., ed. Spin
Labeling-Theory and Applications. Vol. I, pp. 339 372. New York. Academic Press; 1976.

37. Lepock, J.R.; Cheng, K.; Campbell, F.D.; Kruuv, A. Rotational diffusion of Tempone in the cytoplasm of Chinese hamster lung cells. Biophys. J. 44:405 $412 ; 1983$

38. Mehta, A.; Jarori, G.K.; Kenkare, U.W. Spin-labelling of bovine brain hexokinase. Bulletin of Magnetic Resonance (Abstract). 5:252; 1983.

39. Morse, P.D.; Lusczakoski, D.M.; Simpson, D.A. Internal microviscosity of red blood cells and hemoglobin-free resealed ghosts: A spin-label study. Biochemistry 18:5021-5029; 1979 .

40. Pfeiffer, H. Der translationsanteil der protonen relaxation in wassrigen losungen paramagnetischer ionen. Ann. Phys. (Leipzig) 8:1-8, 1961.

41. Polnaszek, C.F.; Bryant, R.G. Self-diffusion of water at the protein surface: A measurement. $J$, Amer. Chem. Soc. 106:428-429, 1984

42. Sienko, M.J.; Plane, R.A. Chemistry: Principles and properties, pp. 1-76 New York: McGraw-Hill; 1966.

43. Solomon, I. Relaxation processes in a system of two spins. Phys. Rev. 99:559-565; 1955.

44. Stone, T.J.; Buckman, T.; Nardio, P.L.; McConnell, N.M. Spin-labelled biomolecules. Proc. Nat. Acad. Sci. 54:1010-1017; 1965.

45. Weinmann, H.J.; Brasch, R.C.; Press, W.R.; Wesbey, G.E. Characteristics of gadolinium-DTPA complex: A potential NMR contrast agent. $A J R$ 142:619-624; 1984.

46. Wesbey, G.E.; Brasch, R.C.; Engelstad, B.L.; Moss, A.A.; Crooks, L.E.; Brito, A.C. Nuclear magnetic resonance contrast enhancement study of the gastrointestinal tract of rats and a human volunteer using nontoxic oral iron solutions. Radiology 149:175-180; 1983.

47. Wesbey, G.E.; Higgins, C.B.: McNamara, M.T.; Engelstad, B.L.; Lipton, M.J.; Ehman, R.L.; Brasch, R.C.; Lovin, J.D. Effect of Gd-DTPA on the magnetic relaxation times of normal and infacted myocardium. Radiology 153:165-170, 1984.

48. Wesbey, G.E.; Moseley, M.E.; Ehman, R.L. Translational molecular self-diffusion in magnetic resonance imaging. I. Effects on observed spin-spin relaxation. Investigative Radiology 19:484-490, 1984.

49. Wesbey, G.E.; Moseley, M.E.; Ehman, R.L. Translational molecular self-diffusion in magnetic resonance imaging. II. Measurement of the self-diffusion coefficient. Investigative Radiology 19:491-498, 1984.

50. Wolff, S. The sister chromatid exchange test. In Stich H.F.; San, R.H.C.; eds. Short term tests for chemical carcinogens, pp. 235-242.

51. Young, I.R.; Bailes, D.R.; Burl, M.; et al. Initial clinical evaluation of a whole body nuclear magnetic resonance (NMR) tomograph. Journal of Computer Assisted Tomography 6:1-18; 1982. 